\title{
Duty cycle tolerant binary gratings for fabricable short period phase masks
}

\author{
E. Gamet \\ emilie.gamet@univ-st-etienne.fr
}

F. Pigeon

O. Parriaux

\author{
Université de Lyon, F-42023, Saint-Etienne, France \\ CNRS, UMR 5516, Laboratoire Hubert Curien, F-42000, Saint-Etienne, France \\ Université de Saint-Etienne, Jean-Monnet, F-42000, Saint-Etienne, France \\ Université de Lyon, F-42023, Saint-Etienne, France \\ CNRS, UMR 5516, Laboratoire Hubert Curien, F-42000, Saint-Etienne, France \\ Université de Saint-Etienne, Jean-Monnet, F-42000, Saint-Etienne, France \\ Université de Lyon, F-42023, Saint-Etienne, France \\ CNRS, UMR 5516, Laboratoire Hubert Curien, F-42000, Saint-Etienne, France \\ Université de Saint-Etienne, Jean-Monnet, F-42000, Saint-Etienne, France
}

Wavelength scale $1 \mathrm{D}$ binary gratings of rectangular corrugation profile are often used as diffractive elements acting on incident free space waves under different incidence angle, wavelength and polarization. Their optical function is best understood by considering the interplay of the grating modes propagating up and down the periodic walls and slits of the segmented structure. The interference conditions between modes depend on the difference between the effective index of the interfering modes and on their relative amplitude. This difference and relative amplitude depend critically on the ratio between the wall and slit widths which is difficult to control technologically. The condition for a wide tolerance of the effective index difference and for a balanced mode excitation on the wall/slit ratio is found analytically and once for all for a wide class of $1 \mathrm{D}$ gratings. It is also found that TE interference elements may exhibit a very wide wall/slit ratio tolerance domain. [DOI: 10.2971/jeos.2009.09047]

Keywords: gratings, phase masks, modal method

\section{INTRODUCTION}

One-dimensional binary gratings of rectangular corrugation profile defined at the surface of a transparent substrate or in a high index film deposited on a substrate exhibit very interesting characteristics which were not expected at the early times of grating coupled integrated optics [1] despite their structural simplicity. It is with the advent of photonic crystals [2] in pace with the development of microelectronics microstructuring technologies [3] that the scientific and application potentials of high aspect ratio and high index contrast periodic structures was realized $[4,5]$. Baets et al. [6] found that a TM beam incident normally from a high index medium onto a binary grating experiences $100 \%$ reflection provided all diffracted orders in the transmission medium are cut off. This mechanism was used successfully in polarization selective laser reflectors generating the radial polarization mode [7]. Mawet et al. [8] found that subwavelength transmission gratings of high index contrast permit to obtain a very wide band $\pi$-phaseshifter. Chang-Hasnein et al. [9] showed that a segmented high index layer deposited on top of a low index substrate can give rise to quasi-100\% reflection of a TM-polarized plane wave normally incident from the air side over a very wide spectral range. All these properties, and the structures exhibiting them, were discovered by numerical modelling, applying the FMM, also called RCWA-method [10] which is reliable if there are no metallic parts in the corrugation [11]. Such method however does not give a hint as to the interplay of the eigenmodes of the segmented structure which is at the basis of all the disclosed effects.

Tishchenko first generalized the modal phenomenology and the related true-mode method [12] for the analysis and synthesis of these infinite periodic waveguides. The modes propagating up and down the periodic grating walls and slits obey the known dispersion equation first given as early as 1956 by Rytov [13]. Clausnitzer et al. [14]-[16] extended the phenomenological modal approach for the understanding of the operation of transmission gratings as grating compressors, laser cavity couplers, and for a more intelligible description of their properties. Gamet et al. [17] also gave a phenomenological interpretation of a novel type of phase masks and a modal understanding of the operation of the GIRO mirror [7].

Whereas most papers dealing with the modal phenomenology of binary gratings address the issue of the optical function, the present paper takes it up in the objective of addressing the issue of fabricability. The operation of most wavelength scale binary grating devices relies upon the interference between two grating modes. Under normal incidence as considered in the present paper, the two modes involved are the even modes of $0^{\text {th }}$ and $2^{\text {nd }}$ order, the $1^{\text {st }}$ order mode having zero field overlap with the incident plane wave. If the index contrast between the grating walls and the low index adjacent media is not too large, the reflection of each mode into itself, as well as the cou- 
pling between the two even modes at the top and bottom of the grating region, is small and can be neglected in the present analysis. It is only in the presence of semiconductor walls and air slits that the modes can be given a second round trip in the grating region as shown in [17]. In the case of low and moderate index contrast, the transmission through the grating is analog to the transmission through a Mach-Zehnder interferometer where the two arms of different length and identical propagation medium are here the two optical paths of the $0^{\text {th }}$ and $2^{\text {nd }}$ modes of identical propagation length and different effective index. The beam splitter and mixer of possibly imbalanced splitting ratio are here the top and bottom interfaces between the grating region and the substrate and cover media where plane waves and grating modes couple with a possible imbalance between modes. The interference conditions between modes depend on the difference between the effective index of the interfering modes and on their relative amplitude. This difference and relative amplitude depend critically on the ratio between the wall and slit widths which is very difficult to control technologically since the period is of the order of the wavelength. These opto-geometrical parameters are all involved explicitly in the dispersion equation which gives the effective index of each mode from where the modal field, therefore the coupling coefficient, can be calculated analytically. A high contrast interference in the transmission medium requires grating modes of equal amplitude, i.e., a tight control of the wall/slit ratio. A prescribed phase shift, $\pi$ for instance, between the two propagated modes in a grating region of given depth, requires also a very accurate control of the wall/slit ratio. Therefore it is an essential task assigned to the designer to find the grating structure exhibiting balanced mode amplitude and prescribed effective index difference over a wide range of wall/slit ratio. The condition for a wide tolerance of the effective index difference and for a balanced mode excitation on the wall/slit ratio will here be searched for analytically from the dispersion equation written in the most normalized form by ultimately reducing the number of independent opto-geometrical parameters. The result will be a universal table of normalized parameters ensuring the desired tolerance condition once for all and for all possible grating structures except those of very high contrast index.

\section{NORMALIZED ANALYSIS OF THE GRATING MODES}

The analysis will here be made for TE modes whose dispersion equation is [12],

$$
\begin{aligned}
& \cos \left(k_{1} r\right) \cdot \cos \left(k_{2}(\Lambda-r)\right)- \\
& \frac{1}{2}\left(\frac{k_{1}}{k_{2}}+\frac{k_{2}}{k_{1}}\right) \cdot \sin \left(k_{1} r\right) \cdot \sin \left(k_{2}(\Lambda-r)\right)=\cos \left(k_{y} \Lambda\right)
\end{aligned}
$$

where, as illustrated in Figure $1, \Lambda$ is the grating period, $r$ is the width of the high index walls, $k_{1}=k_{0}\left(n_{1}^{2}-n_{e}^{2}\right)^{1 / 2}$ and $k_{2}=k_{0}\left(n_{2}^{2}-n_{e}^{2}\right)^{1 / 2}$ express the exponential dependence versus the lateral coordinate $y$ of the electric field of the mode of effective index $n_{e}$ in the walls of high refractive index $n_{1}$ and in the slits of low index $n_{2}$, respectively. $k_{0}=2 \pi / \lambda$ is the spatial frequency of a free space wave in vacuum at wavelength $\lambda . k_{y}=+1$ in case of normal incidence on the grating region. The TE polarization is considered here as the main

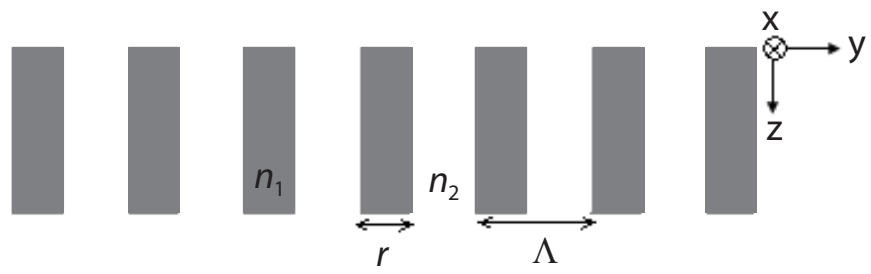

FIG. 1 Section of grating walls and slits of high and low index $n_{1}$ and $n_{2}$, wall width $r$ and period $\Lambda$.

interest of the present study is in the design and fabrication of phase masks. The TE polarization ensures that the contrast of the interferogram produced by the phase mask can possibly be $100 \%$ whatever the angle of the interfering transmitted $+1^{\text {st }}$ and $-1^{\text {st }}$ orders whereas the TM-polarized interferogram exhibit contrast fading (the TM interference contrast reaches zero when the $\pm 1^{\text {st }}$ order diffraction angle is 45 degrees in the transmission medium).

Eq. (1) is purely real and remains real even when $n_{e}>n_{2}$ in which case it becomes,

$$
\begin{aligned}
& \cos \left(k_{1} r\right) \cdot \cosh \left(\left|k_{2}\right|(\Lambda-r)\right)- \\
& \frac{1}{2}\left(\frac{k_{1}}{\left|k_{2}\right|}-\frac{\left|k_{2}\right|}{k_{1}}\right) \cdot \sin \left(k_{1} r\right) \cdot \sinh \left(\left|k_{2}\right|(\Lambda-r)\right)=+1 .
\end{aligned}
$$

There are in Eq. (1) no less than 5 free parameters: $\lambda, n_{1}, n_{2}, \Lambda$, and $r$. Each set of 5 parameters determines a set of eigenmodes having their effective index $n_{e}$. We will here only consider $\mathrm{z}-$ propagating modes of real effective index, leaving the evanescent modes aside. Moreover, we make the assumption that the $0^{\text {th }}$ and $2^{\text {nd }}$ order modes are propagating, that the higher order even mode is sufficiently far from cutoff and that the grating is deep enough to prevent any power transmission through the grating region by evanescent tunnelling of the $4^{\text {th }}$ order mode.

The normalized parameters are defined as follows: $D=$ $r / \Lambda<1$ is the duty cycle of a grating period. $c=n_{2} / n_{1}<1$ is the index contrast between slots and walls. $p=\Lambda /\left(\lambda / n_{1}\right)$ is the ratio between the period and the wavelength in the high index walls. The number of free parameters reduces from 5 to 3. A normalized effective index $v$ is defined as $v=n_{e} / n_{1}<1$. With the above normalization definitions, Eq. (1) becomes

$$
A-\frac{1}{2} B C-1=0
$$

where

$$
\begin{aligned}
& A=\cos \left(2 \pi p D \sqrt{1-v^{2}}\right) \cos \left(2 \pi p(1-D) \sqrt{c^{2}-v^{2}}\right), \\
& B=\left[\left(1-v^{2}\right) /\left(c^{2}-v^{2}\right)\right]^{1 / 2}+\left[\left(c^{2}-v^{2}\right) /\left(1-v^{2}\right)\right]^{1 / 2}, \\
& C=\sin \left(2 \pi p D \sqrt{1-v^{2}}\right) \sin \left(2 \pi p(1-D) \sqrt{c^{2}-v^{2}}\right) .
\end{aligned}
$$

The modal field of each mode is given by the expression

$$
E_{j}(y)=\left\{\begin{array}{cc}
a_{1} \cos \left(k_{1 j}(y-r / 2)\right) & 0<y<r \\
a_{2} \cos \left(k_{2 j}(y-(\Lambda+r) / 2)\right) & r<y<\Lambda
\end{array}\right.
$$

where the $k_{i j}$ terms containing the effective index satisfy the dispersion equation of mode $j$ and $a_{j}$ are amplitude coefficients to be determined at the groove-wall boundary $y=r$, 


$$
a_{1}=a_{2} \frac{\cos \left(k_{2 j}(\Lambda-r) / 2\right)}{\cos \left(k_{1 j} r / 2\right)}
$$

Each field is normalized by dividing Eq. (4) by the integral of the modal field square modulus over one period,

$$
e_{j}(y)=\left\{\begin{array}{cl}
a_{1} \frac{\cos \left(k_{1 j}(y-r / 2)\right)}{\left[\frac{1}{\Lambda} \int_{0}^{\Lambda} E_{j}(y) E_{j}^{*}(y) d y\right]^{1 / 2}} & 0<y<r \\
a_{2} \frac{\cos \left(k_{2 j}(y-(\Lambda+r) / 2)\right)}{\left[\frac{1}{\Lambda} \int_{0}^{\Lambda} E_{j}(y) E_{j}^{*}(y) d y\right]^{1 / 2}} & r<y<\Lambda
\end{array}\right.
$$

Note that the corresponding normalized field expressions can be written as follows by introducing a new dimensionless variable $u=y / \Lambda$. For $0<u<D$,

$$
\begin{aligned}
e_{j}(u)=a_{2} \frac{\cos \left(2 \pi p \sqrt{C^{2}-v_{j}^{2}}(1-D) / 2\right)}{\cos \left(2 \pi p \sqrt{1-v_{j}^{2}} D / 2\right)} & \frac{\cos \left(2 \pi p \sqrt{1-v_{j}^{2}}(u-D / 2)\right)}{\left[\int_{0}^{1} E_{j}(u) E_{j}^{*}(u) d u\right]^{1 / 2}},
\end{aligned}
$$

and for $D<u<1$,

$$
e_{j}(u)=a_{2} \frac{\cos \left(2 \pi p \sqrt{C^{2}-v_{j}^{2}}(u-(D+1) / 2)\right)}{\int_{0}^{1} E_{j}(u) E_{j}^{*}(u) d u} .
$$

\section{THE SEARCH FOR A WIDE TOLERANCE ON DUTY CYCLE}

The interference condition in a grating mode interferometer is first determined by the phase difference $\Delta \Phi$ between the two branches: $\Delta \Phi=k_{0} d\left(n_{e 0}-n_{e 2}\right)$ where $\mathrm{d}$ is the depth of the corrugated region. In the expression of $\Delta \Phi$, the wavelength $\lambda=2 \pi / k_{0}$ is fixed, $d$ can usually be obtained quite reproducibly by the control of dry etching conditions and process time; it is the effective index difference which is the most difficult to achieve reproducibly and, for a given set of wall and slit index $n_{1}$ and $n_{2}$, it is a critically dependent function of the duty cycle $D$. The reason is that the duty cycle $D$ (or equivalently the wall/slit width ratio) is the result of one lithographic step (with possible transfer of the photoresist grating into the antireflection coating) and at least one etching step (two etching steps in the case of electron beam writing because of the presence of a charge neutralizing chromium layer with a loss of lateral dimensional accuracy at each step). The effect of $D$ on the mode effective index is inherently contained in dispersion Eq. (1). Requiring a large tolerance of the effective index difference on $\mathrm{D}$ amounts to cancelling the partial derivative of $v_{0}-v_{2}$ relative to $D$,

$$
\frac{\partial\left(v_{0}-v_{2}\right)}{\partial D}=0
$$

As from here on the dispersion equation, Eq. (1), is considered as a function $F(v, D)=0$ with parameters $p$ and $c$. Considering that Eq. (1) is transcendental, the partial derivative of $v$ relative to $D$ is derived as,

$$
\frac{\partial v}{\partial D}=-\frac{\partial F}{\partial D} / \frac{\partial F}{\partial v}
$$

The wide tolerance condition given by Eq. (8) applied to the Eq.(3) of the dispersion equation writes,

$$
\begin{gathered}
\left.\frac{\partial v}{\partial D}\right|_{\text {mode0 }}-\left.\frac{\partial v}{\partial D}\right|_{\text {mode2 }}=-\left.\frac{\frac{\partial F}{\partial D}}{\frac{\partial A}{\partial v}-\frac{1}{2}\left(B \frac{\partial C}{\partial v}+C \frac{\partial B}{\partial v}\right)}\right|_{\text {mode0 }} \\
+\left.\frac{\frac{\partial F}{\partial D}}{\frac{\partial A}{\partial v}-\frac{1}{2}\left(B \frac{\partial C}{\partial p}+C \frac{\partial B}{\partial p}\right)}\right|_{\text {mode2 }}=0
\end{gathered}
$$

where

$$
\begin{aligned}
\frac{\partial F}{\partial D}=2 \pi p\{- & \sqrt{1-v^{2}} \sin \left(2 \pi p D \sqrt{1-v^{2}}\right) \\
& \times \cos \left(2 \pi p(1-D) \sqrt{c^{2}-v^{2}}\right) \\
+\sqrt{c^{2}-v^{2}} & \cos \left(2 \pi p D \sqrt{1-v^{2}}\right) \\
& \times \sin \left(2 \pi p(1-D) \sqrt{c^{2}-v^{2}}\right) \\
-\frac{1}{2}([(1- & \left.\left.\left.v^{2}\right) /\left(c^{2}-v^{2}\right)\right]^{1 / 2}+\left[\left(c^{2}-v^{2}\right) /\left(1-v^{2}\right)\right]^{1 / 2}\right) \\
& \times \sqrt{1-v^{2}} \cos \left(2 \pi p D \sqrt{1-v^{2}}\right) \\
& \times \sin \left(2 \pi p(1-D) \sqrt{c^{2}-v^{2}}\right) \\
-\sqrt{c^{2}-v^{2}} & \sin \left(2 \pi p D \sqrt{1-v^{2}}\right) \\
& \left.\times \cos \left(2 \pi p(1-D) \sqrt{c^{2}-v^{2}}\right)\right\}
\end{aligned}
$$

$$
\begin{aligned}
\frac{\partial A}{\partial v}=2 \pi p v\left\{\frac{D}{\sqrt{1-v^{2}}} \sin \left(2 \pi p D \sqrt{1-v^{2}}\right)\right. \\
\quad \times \cos \left(2 \pi p(1-D) \sqrt{c^{2}-v^{2}}\right) \\
+\frac{1-D}{\sqrt{c^{2}-v^{2}}} \cos \left(2 \pi p D \sqrt{1-v^{2}}\right) \\
\left.\times \sin \left(2 \pi p(1-D) \sqrt{c^{2}-v^{2}}\right)\right\},
\end{aligned}
$$

$$
\begin{aligned}
\frac{\partial C}{\partial v}=2 \pi p v\{ & \sqrt{1-v^{2}} \sin \left(2 \pi p D \sqrt{1-v^{2}}\right) \\
& \times \cos \left(2 \pi p(1-D) \sqrt{c^{2}-v^{2}}\right) \\
+\sqrt{c^{2}-v^{2}} & \sin \left(2 \pi p D \sqrt{1-v^{2}}\right) \\
& \left.\times \cos \left(2 \pi p(1-D) \sqrt{c^{2}-v^{2}}\right)\right\}, \\
\frac{\partial B}{\partial v}= & v \frac{\left(1-c^{2}\right)^{2}}{\left(1-v^{2}\right)^{3 / 2}\left(c^{2}-v^{2}\right)^{3 / 2}} .
\end{aligned}
$$

The wide tolerance condition Eq. (10) permits to express one of the three normalized parameters $D, c, p$ in terms of the other two. We have chosen the index contrast term $c$ and the duty cycle $\mathrm{D}$ as the independent normalized parameters. 
The problem is now expressed as follows: what is the twodimension subset of $\mathrm{c}$ and $\mathrm{D}$ values which satisfy both the dispersion equation, Eq. (1), and the wide tolerance condition, Eq. (10), then what is the related subset of $p$ values, $p(c, D)$, and optionally, what are the effective index $v_{0}(c, D), v_{2}(c, D)$ and effective index difference $\Delta v(c, D)=v_{0}(c, D)-v_{2}(c, D)$ in the structure characterized by the solution parameters $c$, $D$ and $p$. The information on $\Delta v$ is particularly important to make available since it is the data from which the grating depth $d$ is determined for a desired phase shift $\Delta \Phi$ between modes at wavelength $\lambda$.

The exploration of the domain of $c$ and $D$ is limited to the range of relevant values. The index contrast $c$ will range between 0.34 and 0.66 . These limits correspond to a grating made in a silica or glass substrate with air transmission medium and to a grating in a high index metal oxide such as iron oxide with an index of about 2.9 with air transmission medium. Corrugations made in a high index semiconductor are not considered here because, as shown in [17] with a GaAs grating, the transmission through such high index contrast corrugation can not be considered as a single path. One round trip at least must be taken into account as the effects of the reflections at the grating region boundaries are not negligible. A reasonable range of fabricability for the duty cycle $D$ is from 0.2 to 0.8 . The exploration range of $c$ and $D$ must also correspond to relevant values for the dependent parameter $p$ : the period/wavelength ratio must be such that the diffraction orders propagating in the transmission medium of index $n_{2}$ (the same index as that of the grating slits is assumed) are the $-1^{\text {st }}, 0^{\text {th }}$ and $+1^{\text {st }}$ orders at most. This condition makes sure that higher diffraction orders do not spoil the interferogram produced below the grating by introducing spurious fringes. The cutoff condition for the second diffraction order in the transmission medium is $p \leq 2 / c$. Note at this point that in a phase mask application the $0^{\text {th }}$ transmitted order must be suppressed to produce a single spatial frequency interferogram; this will be achieved by balanced destructive interference as discussed in the next section.

\section{THE CONDITION FOR BALANCED MODE AMPLITUDE}

The first condition for high contrast interference in the transmission medium as a result of the beat between the two modes excited in the grating region is a given phase shift between them. The condition was established in the previous section for a wide tolerance of this phase shift on the duty cycle of the binary corrugation. The second condition for high contrast interference in the transmission medium is now the balanced excitation of the two modes from the incident plane wave and a balanced excitation of the transmitted plane wave from the said two modes in analogy with the request for balanced splitter and mixer in a Mach-Zehnder interferometer. This second condition will be taken into account by simply filtering out all $(c, D)$ pairs retained above which do not ensure balanced mode excitation.

Following [17], the transmission coefficient $t_{s j}$ of a normally incident plane wave from a substrate of refractive index $n_{S}$ to TE mode $j$ of effective index $n_{e j}$ is proportional to an impedance mismatch term and to the integral over one grating period of the normalized electric field of mode $i$ times the incident plane wave field amplitude. Similarly, the transmission coefficient $t_{j c}$ of mode $j$ to the normally transmitted $0^{\text {th }}$ diffraction order in the cover medium of index $n_{c}$ is proportional to the same field integral and to the corresponding impedance mismatch term.

The condition for a balanced interferometer under the hypothesis of negligible reflection and mode coupling at the borders of the grating layer is that the product $T_{0}$ of the transmission coefficient $t_{s 0}$ of the normally incident substrate plane wave of field $E_{s}$ to the $\mathrm{TE}_{0}$ grating mode of field $E_{0}(\mathrm{y})$ by the transmission coefficient $t_{0 c}$ of the $\mathrm{TE}_{0}$ mode to the normally transmitted cover plane wave of field $E_{c}, T_{0}=t_{s 0} t_{0 c}$, is essentially equal to the product $T_{2}$ of the above transmission coefficients involving the $\mathrm{TE}_{2}$ mode, $T_{2}=t_{s 2} t_{2 c}$. This writes,

$$
\frac{t_{s 0} t_{0 c}}{t_{s 2} t_{2 c}} \cong 1
$$

The transmission coefficient $t_{s i}$ of the incident plane wave to the $\mathrm{TE}_{i}$ mode is proportional to the field overlap integral $E_{s} \int_{0}^{\Lambda} E_{i}(y) d y$ and to the said impedance mismatch term. In analogy with the expression of the transmission coefficient through a plane interface between two media of index $n_{s}$ and $n_{i}$ under normal incidence, the impedance mismatch term is $2 \sqrt{n_{s} n_{i}} /\left(n_{s}+n_{i}\right)$.

The condition for a balanced interferometer, Eq. (15) becomes

$$
\left[\int_{0}^{\Lambda} e_{0}(y) d y / \int_{0}^{\Lambda} e_{2}(y) d y\right]^{2} Q=1
$$

where

$$
Q=\frac{n_{0}}{\left(n_{s}+n_{0}\right)\left(n_{c}+n_{0}\right)} \frac{\left(n_{s}+n_{2}\right)\left(n_{c}+n_{2}\right)}{n_{2}}
$$

Substituting the normalized parameters in Eq. (16) gives the normalized condition:

$$
\left[\int_{0}^{1} e_{0}(u) d u / \int_{0}^{1} e_{2}(u) d u\right]^{2} Q=1
$$

It is clear that condition, Eq. (18) with the term $Q$ can not be expressed by the sole set of normalized parameters. Eq. (18) contains $n_{c}$ and $n_{s}$ which in specific structures can be different from $n_{2}$ and $n_{1}$. It is only in structures where the substrate material is the same as the line material $\left(n_{s}=n_{1}\right)$ and the cover material is the same as the groove material $\left(n_{c}=n_{2}\right)$ that the index term $Q$ is expressed with the sole normalized parameters and is equal to

$$
Q=\frac{v_{0}\left(1+v_{2}\right)\left(1+v_{2} / c\right)}{v_{2}\left(1+v_{0}\right)\left(1+v_{0} / c\right)}
$$

It is worth noting that the case of a grating embedded in a single material Eq. (18) is a particular case which gives $Q=$ $\left[v_{0}\left(1+v_{2}\right)^{2}\right] /\left[v_{2}\left(1+v_{0}\right)^{2}\right]$.

Such single structure is however not general enough since very often the corrugation of the grating is made in a high index layer deposited on a low index substrate [17]. 
In order to maintain the generality of the present analysis we will skip the $Q$ index term in the condition Eq. (18) and restrict the balance condition as given by the sole field overlap term. We will see in concrete examples that the effect of this approximation is small.

The search for the triplet values which are solution of the twocondition problem is made as follows: a first matrix is created which contains all possible triplets $(c, D, p)$. Then each step of the process consists in filtering the triplets as sketched in Figure 2.

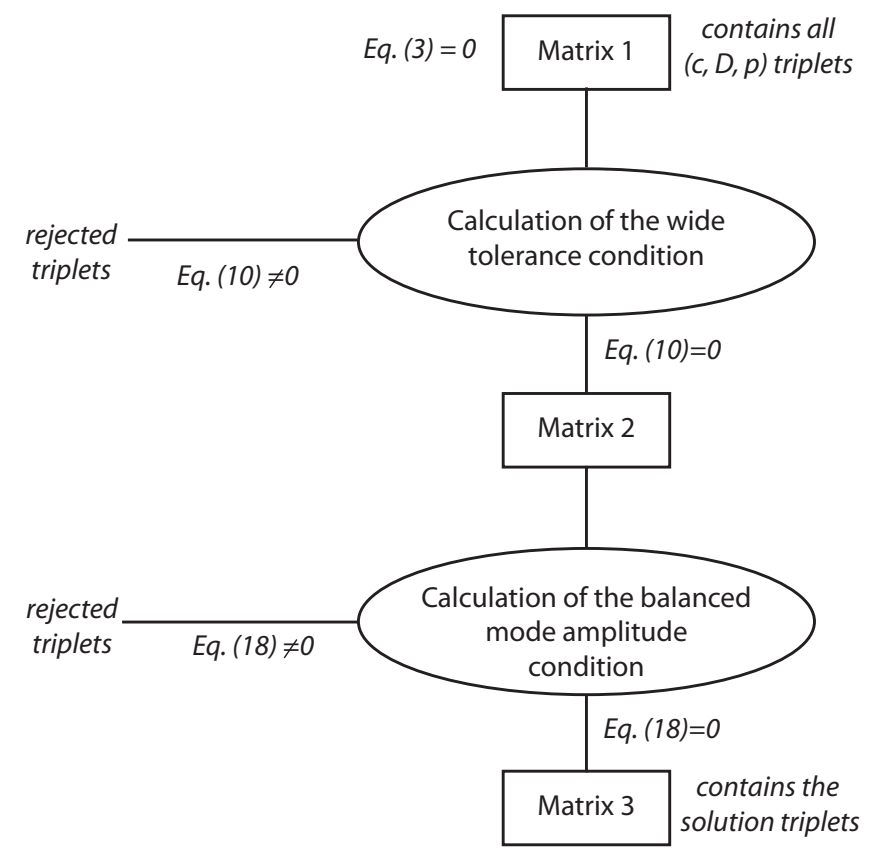

FIG. 2 Search procedure for duty cycle tolerant and balanced two-mode grating interferometers.

\section{SCREENING OF THE STRUCTURAL PARAMETERS FOR TOLERANT HIGH CONTRAST INTERFERENCE}

The most appropriate representation of the normalized structural $(c, D, p)$ parameters satisfying both conditions Eqs. (10) and (18) is in the form of an array. Figure 3 represents the result of a screening with a rather scarce mesh of $c, D$ and $p$ values. It is worth pointing out that Figure 3 is a universal result giving in a normalized form all possible grating structures permitting high contrast interference effects in the transmission medium and large tolerance on the corrugation duty cycle.

A random sampling of the large set of $(c, D, p)$ parameters was made to check on whether these parameters do lead to functional structures exhibiting the desired properties. The functional structure is a binary phase mask whose corrugation is made in a high index film deposited onto a fused quartz substrate with air cover. Usual phase masks intended for fibre Bragg gratings have a period about 4 times larger than the exposure wavelength and are a simple binary corrugation in the fused quartz substrate. Under such condition, the proper choice of the duty cycle and depth of the corrugation leads

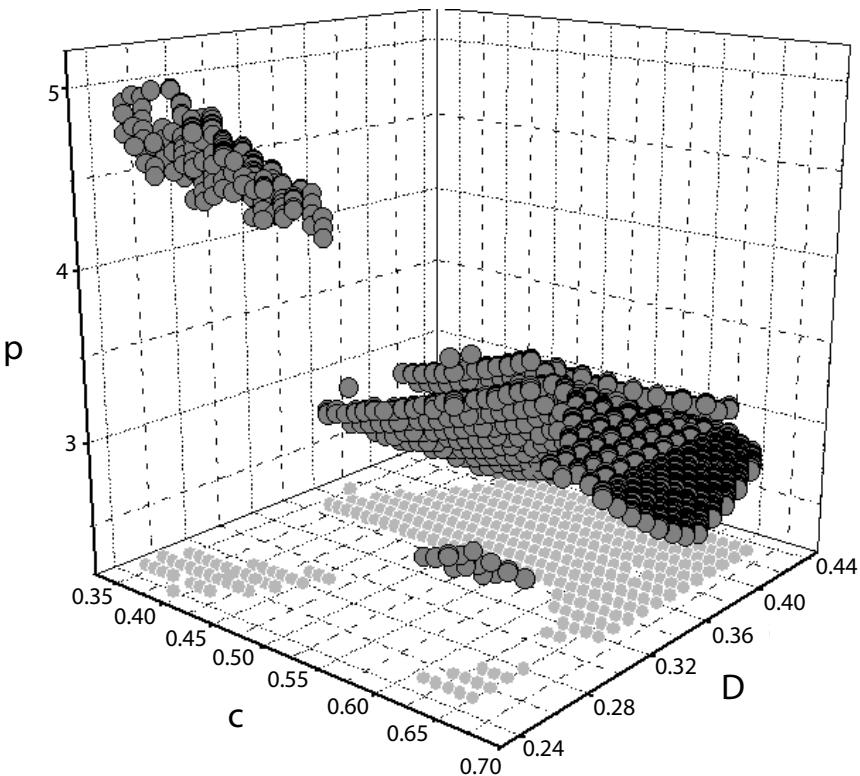

FIC. $33 \mathrm{D}$ distribution of the normalized $(c, D, p)$ parameters defining a line/space tolerant and balanced two mode interferometer. In light gray: projection of the $3 \mathrm{D}$ graph in the $(c, D)$ plane.

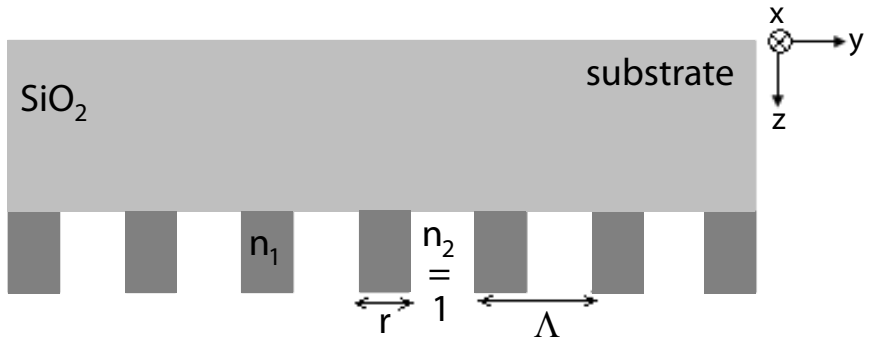

FIG. 4 Short period phase mask with corrugation in a high index layer on silica for tolerant extinction of the $0^{\text {th }}$ transmitted order.

to an extinction of the unwanted $0^{\text {th }}$ diffraction order. In cases where the period and exposure wavelength are of the same order, a standard fused quartz corrugation can not cancel the $0^{\text {th }}$ transmitted order. A corrugation in a high index layer can [17], and this is the optical function on which the structures contained in the chart of Figure 3 will be tested as illustrated in Figure 4.

The substrate is fused quartz and the cover is air $\left(n_{2}=1\right)$. The dimensionless normalized parameters have been converted to actual optogeometrical parameters with $\lambda=700 \mathrm{~nm}$ and $n_{2}=1$. These parameters are given as inputs to an exact vectorial code calculating the diffraction efficiencies. The objective function assigned to the code is the cancellation of the $0^{\text {th }}$ transmitted order in air. It is found that all tested $(c, D, p)$ parameters do lead to the extinction of the $0^{\text {th }}$ transmitted order and to a maximum of the $+1^{\text {st }}$ and $-1^{\text {st }}$ transmitted orders. It is remarkable that the $0^{\text {th }}$ order extinction is actually very tolerant on the duty cycle. This property was further explored and all tested cases have exhibited a quite large tolerance width on the duty cycle $D$ for a $0^{\text {th }}$ transmitted order power smaller than a prescribed threshold. As an example, Figure 5 represents the $0^{\text {th }}$ transmitted order power as well as the first transmitted diffracted order versus the duty cycle $D$ 
in the structure analysed in [17]: a corrugated high index layer of LPCVD $\mathrm{Si}_{3} \mathrm{~N}_{4}$ which is transparent at $325 \mathrm{~nm}$ wavelength $\left(n_{1}=2.12\right)$, a period of $400 \mathrm{~nm}$ on a fused quartz substrate. The corresponding triplet $(c, D, p)$ is $(0.47,0.4,2.6)$ and appears as solution in the final matrix (see Figure 3). Figure 5 shows that the optimised structure exhibits a flat of the $0^{\text {th }}$ order below $1 \%$ over a domain of $D$ between 0.38 and 0.43 . The $0^{\text {th }}$ order actually remains acceptably small (less than $2 \%$ ) over a significantly wider range, especially at the side of narrow high index wall widths. Technologically, a tolerance between about 140 and $175 \mathrm{~nm}$ on the width of the silicon nitride walls in a grating of $400 \mathrm{~nm}$ period is quite comfortable and permits the use of interference lithography instead of electron beam patterning.

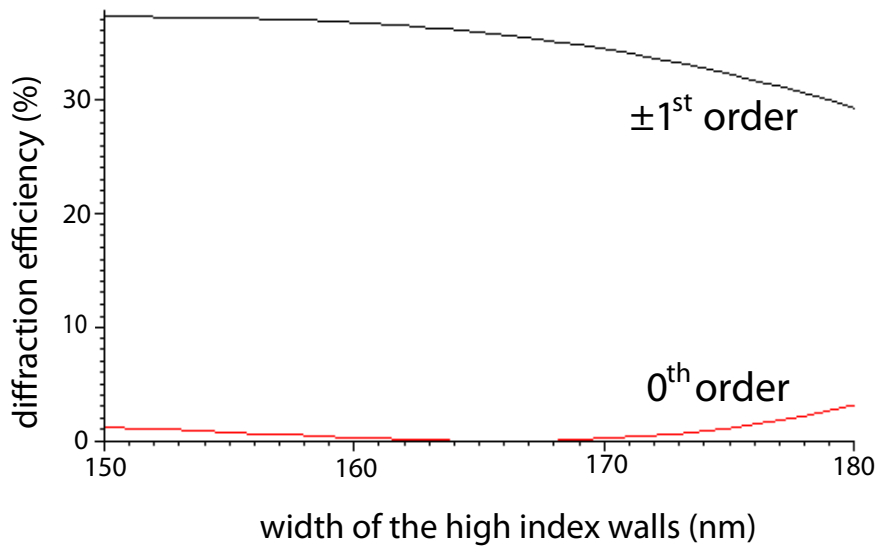

FIG. 5 Diffraction efficiency of the $0^{\text {th }}$ and $1^{\text {st }}$ orders versus the width of the high index wall.

The 3D chart of Figure 3 teaches that all structures allowing the cancellation of the $0^{\text {th }}$ transmitted order have a duty cycle $D$ smaller than 0.45 . The effective index and field dependences on the duty cycle when $D$ is larger than 0.5 become sharper, especially for the $\mathrm{TE}_{2}$ mode whose electric field distribution changes significantly upon a variation of $D$. There are three clouds of possible operation points $(c, D, p)$ in the chart. Two clouds correspond to a particularly small duty cycle of about 0.25 and to high and small index contrast $(c=0.38$ and 0.64 respectively). In the small duty cycle and large index contrast cloud and in the small duty cycle and low index contrast cloud a variation of $D$ does not impose strong variation of the modal field and of the effective index of the $\mathrm{TE}_{0}$ and $\mathrm{TE}_{2}$ modes as the boundaries are far from each other. The main cloud encompasses points corresponding to a larger duty cycle. There are a large number of structures which enable a cancellation of the $0^{\text {th }}$ transmitted order between 0.34 and 0.43 and the index contrast is practically unlimited except at the high contrast side. These 3 zones correspond to different variation domains of $p$. The main cloud and the duty cycle and large index contrast cloud correspond to the lower $p$ values $(2.24<p<3.49)$ and the last one to large normalized periods $(4.20<p<5.02)$.

\section{CONCLUSION}

The normalized analysis of a phase mask structure of period of the order of the wavelength, whose binary corrugation is fabricated in a high index layer deposited on a low index substrate, operating with the TE polarization under normal inci- dence can be designed with a wide choice of possible index contrasts and a not too restricted choice of duty cycles below 0.43 . This means that the variety of high index layer materials that can be used is only limited by their transparency at the exposure wavelength. The two conditions leading to a destructive interference of the $0^{\text {th }}$ order transmission (local independence of the effective index difference on the duty cycle and equality of the modal field overlap) have been expressed analytically and permit to express them in a universal 3D chart containing all possible solutions. A remarkable characteristics of the operation points identified is that the condition for zero $0^{\text {th }}$ transmitted order is very little dependent on the duty cycle: not only the derivative of the effective index difference cancels, but it does so over a very wide range of $D$ which eases considerably the fabrication conditions of the needed short period binary gratings. This means, in particular, that such phase masks of wavelength scale period do not have to be fabricated by electron beam pattern generators, but mask photolithography and even interferogram projection can be used, thanks to the large tolerance obtained by the present analysis. The reason for such wide tolerance may be due to the two conditions (effective index difference and equality of mode coupling) being somehow related. Further work is needed to elucidate this issue.

\section{References}

[1] T. Tamir, "Beam and waveguide couplers" in Integrated Optics, T. Tamir, ed., pp.83-137 (Springer-Verlag, New York, 1985)

[2] T. F. Krauss, R. M. Delarue, and S. Brand, "Two-dimensional photonic-bandgap structures operating at near-infrared wavelengths" Nature 383, 699-702 (1996).

[3] T. C. Kleckner, D. Modotto, A. Locatelli, J. P. Mondia, S. Linden, R. Morandotti, C. D. Angelis, C. R. Stanley, H. M. van Driel, and J. S. Aitchison, "Design, Fabrication, and Characterization of DeepEtched Waveguide Gratings" J. Lightwave Technol. 23, 3832-3842 (2005).

[4] D. K. Jacob, S. C. Dunn, and M. G. Moharam, "Design considerations for narrow-band dielectric resonant grating reflection filters of finite length" J. Opt. Soc. Am. A 17, 1241-1249 (2000).

[5] E. Bonnet, A. Cachard, A. V. Tishchenko, and 0. Parriaux, "Scaling rules for the design of a narrow-band grating filter at the focus of a free-space beam" Proc. SPIE 5450, 217 (2004).

[6] S. Goeman, S. Boons, B. Dhoedt, K. Vandeputte, K. Caekebeke, P. van Daele, and R. Baets, "First demonstration of highly reflective and highly polarizationselective diffraction gratings (GIROgratings) for long-wavelength VCSELs" IEEE Photonic. Tech. L. 10, 1205-1207 (1998).

[7] T. Moser, J. Balmer, D. Delbeke, P. Muys, S. Verstuyft, and R. Baets, "Intracavity generation of radially polarized $\mathrm{CO}_{2}$ laser beams based on a simple binary dielectric diffraction grating" App. 0pt. 45, 8517-8522 (2006).

[8] D. Mawet, P. Riaud, J. Surdej, and J. Baudrand, "Subwavelength surface-relief gratings for stellar coronagraphy" Appl. Opt. 44, 7313-7321 (2005).

[9] C. F. R. Mateus, M. C. Y. Huang, Y. Deng, A. R. Neureuther, and C. Chang-Hasnain, "Ultrabroadband mirror using low-index cladded subwavelength grating" IEEE Photonic. Tech. L. 16, 518520 (2004). 
[10] P. Lalanne, and G. M. Morris, "Highly improved convergence of the coupled-wave method for TM polarization" J. Opt. Soc. Am. A 13, 779-784 (1996).

[11] N. M. Lyndin, 0. Parriaux, and A. V. Tishchenko, "Modal analysis and suppression of the Fourier modal method instabilities in highly conductive gratings" J. Opt. Soc. Am. A 24, 3781-3788 (2007).

[12] A. V. Tishchenko, "'Phenomenological representation of deep and high contrast lamellar gratings by means of the modal method" Opt. Quant. Electron. 37, 309-330 (2005).

[13] S. M. Rytov, "Electromagnetic properties of a finely stratified medium" Sov. Phys. JETP-USSR 2, 466-475 (1956).

[14] T. Clausnitzer, T. Kämpfe, E.-B. Kley, A. Tünnermann, A. V. Tishchenko, and 0. Parriaux, “Highly-dispersive dielectric transmission gratings with $100 \%$ diffraction efficiency" Opt. Express $16,5577-5584$ (2008).

[15] T. Clausnitzer, T. Kämpfe, E.-B. Kley, A. Tünnermann, U. Peschel, A. V. Tishchenko, and 0. Parriaux, "An intelligible explanation of highly-efficient diffraction in deep dielectric rectangular transmission gratings" Opt. Express 13, 10448-10456 (2005).

[16] A. Bunkowski, 0. Burmeister, K. Danzmann, R. Schnabel, T. Clausnitzer, E.-B. Kley, and A. Tünnermann, "Demonstration of threeport grating phase relations" Opt. Lett. 31, 2384-2386 (2006).

[17] E. Gamet, A. V. Tishchenko, and 0. Parriaux, "Cancellation of the zeroth order in a phase mask by mode interplay in a high index contrast binary grating" Appl. 0pt. 46, 6719-6726 (2007). 